preventive strategies should be initiated to curb this rising prevalence of allergies.

\section{WHAT DO CHILDREN WITH CONGENITAL HEART DISEASES DO WHEN THEY GROW UP}

doi:10.1136/archdischild-2012-302724.0450

SC Antoniadis, A Antoniadou, O Panagiotopoulos, E Milopteri, P Kleanthous, E Dionyssopoulou. Diagnostic Center for Pediatric Cardiology, Athínai, Greece

Background Advances in the diagnosis and interventional-surgical management of congenital heart diseases had as a result a considerable number of patients reaching the adolescent and young adult life.

Aims The aim of this study was to investigate the influence of the heart problem on the psychosocial-sexual and family life, careers and employment rate of patients with congenital heart disease.

Methods We studied according to a protocol with a closed questionnaire as well as the medical files of 441 children with congenital heart diseases.

Results From the 158 male patients aged $15-35$ years, 98(62\%) had simple heart lesions, 21(13.3\%) combinations of simple heart lesions and 39(24.7\%) complex heart problems, 93(59\%) had surgicalinterventional management. Between them 130(82.2\%) had no psychosocial-sexual problems related with age, whereas $13(8.3 \%)$ with complex heart lesions had not normal social and sexual life. From the 146 female patients aged $14-32$ years, 93(63.7\%) had simple lesions, $25(17.12 \%)$ combinations of simple heart lesions, and $28(19.17 \%)$ complex heart problems, $56(38.3 \%)$ had surgicalinterventional management. From them $136(93.15 \%)$ had no problems related with age whereas $10(6.85 \%)$ had, from the 23 pregnancies, 10(43.5\%) operated mothers, 21(91.3\%) babies born without problems. From the 114 patients studied for their careers and employment rate, $59(59.8 \%)$ were high school graduates, $43(37.7 \%)$ university graduates, $9(7.9 \%)$ technical school graduates, $103(90.4 \%)$ were employed, 11(9.6\%) couldn't work because of the heart problem.

Conclusions Simple heart lesions and their combinations doesn't cause specific problems whereas complex heart diseases, especially if the result of the operation is not the expected one, cause.

\section{THE EFFECT OF PRETERM BIRTH ON ATTENTIONAL NETWORKS IN ADLULTS}

doi:10.1136/archdischild-2012-302724.0451

A von Muhlenen, N Baumann, D Wolke. University of Warwick, Coventry, UK

Background Preterm birth has been associated with an increased risk of cognitive, behavioral and psychiatric problems. In this study we examine how these problems are linked to specific attentional networks.

Methods Based on a sample from the Bavarian Longitudinal Study we compared a cohort of 97 survivors born very preterm $(<32$ weeks gestational age, GA) or very-low-birth-weight (VLBW < $1500 \mathrm{~g}$ ) in 1985/86 (mean birth weight, 1318g; mean GA at birth, 30.2 weeks; $52 \%$ male) with 109 controls from the same population in Bavaria (mean birth weight, 3414g; mean GA at birth, 39.7 weeks; $48 \%$ male). We looked at performance in the attention network test, developed by Fan et al. (2002, Journal of Cognitive Neuroscience), in three anatomically defined attentional networks: alerting, orienting and executive control.

Results The figure shows the RT index, representing efficiency for each attentional network. The VLBW group showed a large and highly significant deficit in the executive network and a smaller but significant deficit in the orienting network, but no deficit in the alerting network. Although RTs were generally longer for VLBW, the RT index result did not change when using RT ratio scores.

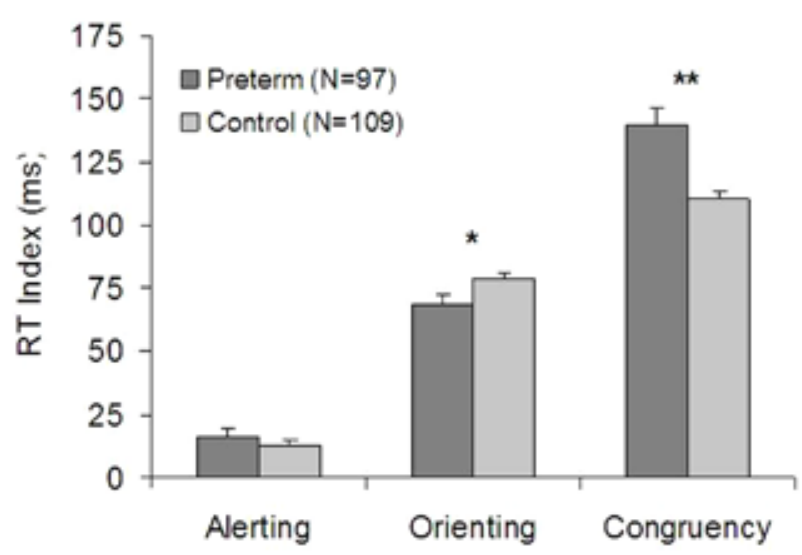

Attentional Network

Abstract 451 Figure 1 RT Index

Conclusions The results show that VLBW is linked to specific attentional deficiencies, especially those involving executive control. These results are discussed in the light of the literature on VLBW and attention.

\section{ASSOCIATIONS BETWEEN BEHAVIORAL AND SLEEPING DISORDERS IN PRETERM CHILDREN TO CATCH UP IN HEIGHT AND WEIGHT}

doi:10.1136/archdischild-2012-302724.0452

${ }^{1}$ E Deschman, ${ }^{1} \mathrm{M}$ Vanpee, ${ }^{1} \mathrm{~L}$ Legnevall, ${ }^{2} \mathrm{~A}$ Kistner. ${ }^{1}$ Inst of Women and Child Health; 2Inst of Molecular Medicine and Surgery, Stockholm, Sweden

Aim To study an association between sleeping (SP) and behavioural disorders (BP) in preterm children to catch-up growth and weight.

Design and Methods 105 children (8.5-10 years): 39 born preterm (<32 weeks), at term 30 born SGA and 36 born AGA (Controls) were studied. Eight Preterms were born SGA and 31 were born AGA. BP was defined as hyperactivity and concentration problems, SP as hard to fall asleep and wakening during night. Pearson Chi-Square or Student t-test were used.

Results The Preterm group displayed a higher incidence of SP $(23 \%$ vs $10 \%$ and $3 \% \mathrm{p}=0.026)$ and $\mathrm{BP}(38 \%$ vs $10 \%$ and $8 \%, \mathrm{p}=0.001)$.

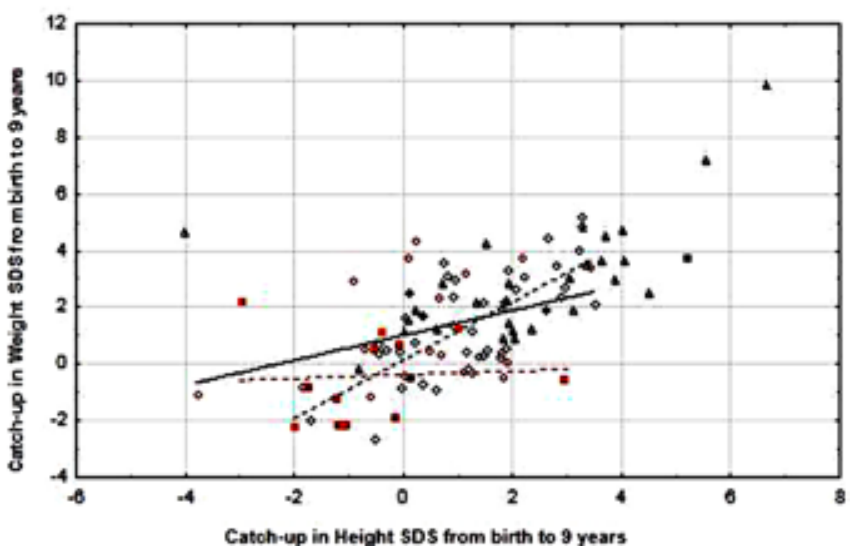

Abstract 452 Figure 1 BP in relation to catch up growth

o Preterm AGA no BP•Preterm AGA with BP $\diamond$ Control no BP 
The black dotted line: Controls without BP $(r=0.69, p<0.0001)$. The solid line: Preterm AGA without BP $(r=0.38, p=0.11)$, the red dotted: preterms AGA with $B P(r=0.06, p=N S)$.

At 9 years, preterm AGA with BP $(n=13)$ had lower length SDS $(p=0.003)$, weight SDS $(p=0.006)$ and head circumference SDS and a tendency to lower height catch-up $(p=0.09)$ compared to preterm AGA without BP $(n=18)$. Fasting levels of IGF-I, insulin and leptin were lower in all Preterms with BP.

Preterms with SP $(n=8)$ had a lower height catch-up $(\mathrm{p}=0.009)$ compared to those without SP $(\mathrm{n}=30)$.

Conclusion Children born preterm have an increased risk for SP and BP. These disorders are associated with reduced catch up in height.

\section{REPEAT COURSES OF ANTENATAL CORTICOSTEROIDS FOR PRETERM BIRTH AND RISK FOR METABOLIC SYNDROME IN YOUNG ADULTHOOD}

doi:10.1136/archdischild-2012-302724.0453

${ }^{1} \mathrm{H}$ Norberg, ${ }^{2} \mathrm{~J}$ Stålnacke, ${ }^{2} \mathrm{R}$ Diaz Heijtz, ${ }^{3} \mathrm{AC}$ Smedler, ${ }^{2} \mathrm{H}$ Forssberg, ${ }^{1} \mathrm{M}$ Norman. ${ }^{1}$ Department of Clinical Science, Intervention and Technology; ${ }^{2}$ Department of Women's and Children's Health, Karolinska Institutet; ${ }^{3}$ Department of Psychology, Stockholm University, Stockholm, Sweden

Background Preterm birth is associated with later hypertension and diabetes. One explanation for this association could be that exposure to antenatal corticosteroids (ACS), especially if repeated, induce adverse long-term effects. There are no data on whether repeat courses of ACS are associated with health problems later in life. The aim of this study was to assess whether repeat courses of ACS correlate to metabolic syndrome later in life.

Methods In a population-based cohort we measured BMI, blood pressure, arterial stiffness, blood lipids and glucose tolerance in 58 subjects ( 36 boys, age 14 to 26 years) exposed to 2-9 weekly courses of antenatal betamethasone. Subjects exposed to a single course ( $n=25,14$ boys) and unexposed subjects ( $n=44,25$ boys) were included as comparison groups.

Results As compared to unexposed controls, subjects exposed to repeat courses of ACS did not differ in BMI (mean difference $0.6 \mathrm{~kg} /$ $\mathrm{m}^{2}, \mathrm{p}=0.5$ ), mean systolic or diastolic blood pressure (mean diff $1 \mathrm{mmHg}, \mathrm{p}=0.78-0.83$ ), arterial stiffness assessed by pulse wave analysis (mean diff $0.1 \%, \mathrm{p}=0.50$ ), triglyceride (mean diff $0.1 \mathrm{mmol} / \mathrm{L}$ ), total cholesterol (mean diff $0 \mathrm{mmol} / \mathrm{L}$ ), LDL/HDL ratio (mean diff 0.1), Lipoprotein(a) (mean diff. $61 \mathrm{mg} / \mathrm{L}$ ), ApolipoproteinB/ApolipoproteinA1 ratio (mean diff 0.01$),(p=0.33-0.91)$ or glucose tolerance assessed by HOMA-index (mean diff $0, \mathrm{p}=0.84$ ). Subjects exposed to a single course of ACS did not differ from the other groups in any of the variables above.

Conclusions Repeat courses of ACS do not correlate to metabolic syndrome in young adulthood. This observation has clinical implications for the ongoing discussion about safety of antenatal steroids.

\section{HEPATIC GLYCOGENOSIS IN TYPE I DIABETES MELLITUS: REPORT OF TWO CASES AND REVIEW OF THE LITERATURE}

doi:10.1136/archdischild-2012-302724.0454

LW de Groot, WB Geven. Pediatrics, Martini Hospital, Groningen, The Netherlands

Aim Hepatic glycogenosis is an underrecognized cause of serum transaminase elevations in poorly controlled type I diabetes mellitus, which has a relatively benign course with appropriate treatment. ${ }^{1,2}$ Objective of this study is to describe the aetiology, clinical presenting symptoms and treatment options.

Methods A report of two adolescents with poor controlled diabetes mellitus, hepatomegaly and serum transaminase elevations and a literature review.
Results Both cases presented with abdominal pain and hepatomegaly, combined with nausea and dyspeptic complaints. Laboratory investigation revealed marked elevation of serum transaminase levels. Synthetic function of the liver stayed intact. Abdominal ultrasound showed isolated, homogenous hepatomegaly, without other abdominal abnormalities. In one case liver biopsy was performed, showing hepatic glycogenosis. Other causes for hepatomegaly were excluded. With improved diabetic control all complaints improved within three weeks, with normalisation of serum transaminase levels.

Review of literature that hepatic glycogenosis, not frequently described, is an important complication of type I diabetes mellitus. Hepatic glycogenosis as result of glycogen storage in hepatocytes, caused by periods of hyperglycaemia and frequent insulin boluses. This process is reversible with improved glycaemic control. ${ }^{1,2}$

Conclusions Hepatic glycogenosis is a important complication of type I diabetes mellitus which can be reversible with the proper treatment. Therefore, medical attention is necessary.

\section{References}

1. C.F.J. Munns et al. Hepatic glycogenosis: Reversible hepatomegaly in type 1 diabetes. Journal of Paediatrics and Child Health 2000; 36:449-452.

2. M. Torbenson et al. Glycogenic Hepatopathy, an underrecognized hepatic complication of diabetes mellitus. The American Journal of Surgical Pathology 2006; 30:508-513.

\section{IMPROVEMENT OF SERUM TESTOSTERONE IN DIABETIC RATS TREATED WITH METFORMIN AND NIGELLA SATIVA}

doi:10.1136/archdischild-2012-302724.0455

${ }^{1} \mathrm{~A}$ Abd ElShaheed, ${ }^{2} \mathrm{KA}$ El-Shamy, ${ }^{2} \mathrm{TH}$ Mekhael, ${ }^{3} \mathrm{~F}$ Adly, ${ }^{4} \mathrm{R}$ Boulos, ${ }^{4} \mathrm{SE}$ Ibrahim, ${ }^{2} \mathrm{NN}$ Fadl. ' $\mathrm{C}$ Child Health; ${ }^{2}$ Physiology; ${ }^{3}$ Pathology, National Research Centre; 'Zoology Department, Faculty of Science, Cairo University, Cairo, Egypt

Background and Aims To evaluate the effect of metformin and Nigella sativa (alone or in combination) on improving the diabetic state of rats.

Methods Male Sprague-Dawley rats weighing 180-200g had induced diabetes using alloxan $(150 \mathrm{mg} / \mathrm{kg})$, then diabetic rats were treated daily for 45 days with metformin $(0.5 \mathrm{~g} / \mathrm{Kg}$.b.wt), Nigella sativa $(1 \mathrm{~g} / \mathrm{Kg} . b . w t)$ or a mixture of metformin + Nigella sativa $(0.25 \mathrm{~g}+1 \mathrm{~g} / \mathrm{Kg}$.b.wt $)$ in a separated three groups and compared with a group of alloxanated diabetic rats as control. HbA1c, serum glucose, lipid profile, microalbuminuria (MA), ALT, AST, insulin, SHBG and total testosterone were measured using ELISA \& spectrophotometer techniques, testis and liver tissue were examined histopathologically.

Results Both metformin and Nigella sativa were comparable in reducing serum glucose of the diabetic rats, furthermore, Nigella sativa showed a hypolipidaemic effect and it also improved liver functions. The level of serum insulin was significantly increased $(\mathrm{P}<0.05)$ in three groups. Importantly, using the mixture of metformin and N.sativa was less effective in improving diabetic state than using metformin or N.sativa alone, although it had improved serum level of testosterone and normalized the structure of testis.

Conclusion Using either metformin or Nigella sativa alone was more effective in improving the diabetic state of rats than using them in combination, although this combination was more effective in improving both serum level of testosterone and the structure of testis. This raise basic questions about the effect of interactions that may occur on using this mixture in the treatment of diabetes that necessitate further studies.

456 SEGMENTAL ACUTE SPINAL CORD SYNDROME: "AS A BOMB" 